University of Texas at El Paso

ScholarWorks@UTEP

6-1999

\title{
Multi-Resolution Techniques in the Rules-Based Intelligent Control Systems: A Universal Approximation Result
}

Yeung Yam

Hung T. Nguyen

Vladik Kreinovich

The University of Texas at El Paso, vladik@utep.edu

Follow this and additional works at: https://scholarworks.utep.edu/cs_techrep

Part of the Computer Engineering Commons

Comments:

UTEP-CS-99-22.

Published in the Proceedings of the 14th IEEE International Symposium on Intelligent Control/ Intelligent Systems and Semiotics ISIC/ISAS'99, September 15-17, 1999, Cambridge, Massachusetts, USA, pp. 213-218.

\section{Recommended Citation}

Yam, Yeung; Nguyen, Hung T.; and Kreinovich, Vladik, "Multi-Resolution Techniques in the Rules-Based Intelligent Control Systems: A Universal Approximation Result" (1999). Departmental Technical Reports (CS). 560.

https://scholarworks.utep.edu/cs_techrep/560

This Article is brought to you for free and open access by the Computer Science at ScholarWorks@UTEP. It has been accepted for inclusion in Departmental Technical Reports (CS) by an authorized administrator of ScholarWorks@UTEP.For more information, please contact Iweber@utep.edu. 


\title{
Multi-Resolution Techniques in the Rules-Based Intelligent Control Systems: A Universal Approximation Result
}

\author{
Yeung Yam \\ Department of Mechanical \\ \& Automation Engineering \\ The Chinese University of Hong Kong \\ Shatin, NT, Hong Kong, China \\ email yyam@mae.cuhk.edu.hk \\ Hung T. Nguyen \\ Department of Mathematical Sciences \\ New Mexico State University \\ Las Cruces, NM 88003, USA \\ email hunguyen@nmsu.edu \\ Vladik Kreinovich \\ Department of Computer Science \\ University of Texas at El Paso \\ El Paso, TX 79968, USA \\ email vladik@cs.utep.edu
}

\begin{abstract}
Intelligent control is a very successful method of transforming expert knowledge of control rules (formulated in terms of natural language, like "small") into a precise control strategy. It has led to many spectacular applications, ranging from appliances to automatic subway control to super-precise temperature control on a Space Shuttle mission.

It is known that fuzzy control is a universal approximator, i.e., that it can approximate every possible control strategy within an arbitrary accuracy. One of the main problems of fuzzy control is that the number of rules which are necessary to represent a given control strategy with a given accuracy, grows exponentially with the increase in accuracy. As a result, for reasonable accuracy, and a reasonable number of input variable, we sometimes need astronomically many rules.

In this paper, we start to solve this problem by pointing out that traditional one-step fuzzy rule bases, in which expert rules directly express control in terms of the in-
\end{abstract}

put, are often a simplification of the actual multi-step expert reasoning. We show that a natural formalization of such expert reasoning leads to a universal approximation result in which the number of control rules does not increase with the increase in accuracy. Thus, this multi-resolution approach looks like a promising solution to the rule base explosion problem.

\section{Intelligent Control: Rules Explosion as a Main Problem}

Intelligent control is a very successful method of transforming expert knowledge of control rules (formulated in terms of natural language, like "small") into a precise control strategy. During the $25+$ years of its existence, it has led to many spectacular applications, ranging from appliances to automatic subway control to superprecise temperature control on a Space Shuttle mission (see, e.g., $[8,9]$ ).

The fact that intelligent control is a reasonable strategy is emphasized by the results that every possible control 
strategy can be, within an arbitrary accuracy, approximated by a fuzzy controller (for a recent survey of such results, see, e.g., [16, 26]).

The problem with this approach is that the number of rules which are necessary to represent a given control strategy with a given accuracy, grows exponentially with the increase in accuracy. As a result, for reasonable accuracy, and a reasonable number of input variable, we need astronomically many rules (see, e.g., $[10,12,13,15,14])$.

\section{How This Problem is Solved Now}

This problem has been recognized for a quite a while, and several good approaches have been proposed, including approaches by B. Kosko and L. Koczy [10, 12, $13,15,14])$.

Kosko's main idea consists of building the rules around the extrema of the control function. In most practical examples, if a control function has few extrema, then we are able to approximate this control with much fewer rules than in the general case.

Koczy's idea is, crudely speaking, to reduce the number of rules by applying an appropriate transformation of the membership functions.

Both approaches work well in realistic situations when the desired control strategy is reasonably smooth (and slowly changing) outside a few critical regions where the specific strategy is important (see, e.g., [32] and references therein).

\section{A Multi-Resolution Approach to Solving This Problem: Main Idea}

Another possible answer to the exponential increase problem is the multi-resolution approach (see, e.g., $[22,23]$, and references therein). This answer is based on the following observation:

- Traditional fuzzy control technique is based on the assumption that we have the rules which directly relate the input $x=\left(x_{1}, \ldots, x_{n}\right)$ with the control, rules like "if $x_{1}$ is small, ..., and $x_{n}$ is medium, then $u$ should be large".

- In many real-life situations, however, the decision making process of expert human controllers is $h i$ erarchical. When given a set of measured values $x_{1}, \ldots, x_{n}$ which characterize the state of the controlled system (plant), the human controller does not immediately starts a control. The reason for this is that natural control rules are often formulated in terms of some parameters $y_{1}, \ldots, y_{m}$ of the system, which are difficult (or even impossible) to measure directly. Therefore, before the expert controller can come up with a reasonable control value, he has to:

- first, to estimate the values of these parameters $y_{i}$ based on the available knowledge (i.e., on the measured values $x_{j}$ ); and

- only then, based on the values of $y_{i}$, produce a reasonable control $u$.

On both stages, the controller uses "fuzzy" rules.

The main result of this paper is that with such twolevel systems, we can approximate an arbitrary control strategy by a rule base without invoking an unrealistic exponential increase in the number rules. Moreover, we will show that this is true even when on each stage, we have simple fuzzy rules, with a crisp conclusion.

Let us describe such rule bases in precise terms.

\section{Towards a Formal Description of Two-Stage Rule-Based Fuzzy Control}

We want to describe the expert controller's rules, both the first-stage rules, which describe the auxiliary variables $y_{1}, \ldots, y_{m}$ in terms of the inputs $x_{1}, \ldots, x_{n}$, and the second-stage rules, which describe the control $u$ in terms of $y_{j}$.

\subsection{Description of Each Stage: Traditional Approach}

Since we decided to consider only the simplest rules, for which the conclusion is crisp, the general definition of a one-stage rule base will look like this:

Definition 1. Let $N$ be a positive integer, and let $z_{1}, \ldots, z_{N}, t$ be variables. By a rule base which describes $t$ in terms of $z_{i}$, we mean a finite collection $\mathcal{R}$ of rules of the type

$$
\begin{gathered}
\text { "if } z_{1} \text { is } A_{r 1}, z_{2} \text { is } A_{r 2}, \ldots, \text { and } z_{N} \text { is } A_{r N}, \\
\text { then } t=t^{(r)} \text { ", }
\end{gathered}
$$

where $r=1, \ldots, R, A_{r i}$ are fuzzy sets (with continuous membership functions $\left.\mu_{r i}\left(z_{i}\right)\right)$, and $t^{(1)}, \ldots, t^{(R)}$ are real numbers. 
Fuzzy control technique translates these rules into a precise dependence $t=f\left(z_{1}, \ldots, z_{N}\right)$ which is defined as follows:

Definition 2. Let $\mathcal{R}$ be a rule base (1) which describes $t$ is terms of $z_{j}$, and let $f_{\&}$ be a continuous t-norm.

- For each set of values $z_{1}, \ldots, z_{N}$, and for each $r=1, \ldots, R$, by a degree $d_{r}$ with which $r$-th rule is fired, we mean a number $d_{r}=$ $f_{\&}\left(\mu_{r 1}\left(z_{1}\right), \ldots, \mu_{r N}\left(z_{N}\right)\right)$.

- By a function $t=f_{\mathcal{R}}\left(z_{1}, \ldots, z_{N}\right)$ corresponding to the rule base $\mathcal{R}$, we mean a function

$$
t=\frac{d_{1} \cdot t^{(1)}+\ldots+d_{R} \cdot t^{(R)}}{d_{1}+\ldots+d_{R}}
$$

4.2 Description of Each Stage: New Approach Aimed at Decreasing the Number of Rules

Definitions 1 and 2 describe a traditional description of fuzzy rules, in which we have to describe the appropriate value of $t$ for all possible combinations of $z_{i}$. The number of such combinations exponentially increases with the growth of $N$, so, when $N$ increases, the necessary number of rules starts growing exponentially. To somewhat curb this growth, we can use the fact that often, the same value $t$ is reasonable for several different combinations of $z_{i}$. For example, the fact that we use "if-then" rules naturally leads to a possibility of adding, to the rule base (1), an "else" clause

$$
\text { "else } t=t^{(0)} " \text {. }
$$

The degree $d_{0}$ to which this "else" rule is fired can be defined, e.g., as $1-\left(d_{1}+\ldots+d_{R}\right)$. This is possible only if $d_{1}+\ldots+d_{R} \leq 1$; in this case, $d_{1}+\ldots+d_{R}+d_{0}=1$, and the formula (2) becomes even simpler. As a result, we arrive at the following definitions:

Definition $\mathbf{1}^{\prime}$. Let $N$ be a positive integer, and let $z_{1}, \ldots, z_{N}, t$ be variables. By a rule base which describes $t$ in terms of $z_{i}$, we mean a finite collection $\mathcal{R}$ of $R$ rules of type (1) plus an additional "else"-rule (3).

Definition $2^{\prime}$. Let $\mathcal{R}$ be a rule base (1), (3) which describes $t$ is terms of $z_{j}$, and let $f_{\&}$ be a continuous t-norm.

- For each set of values $z_{1}, \ldots, z_{N}$, and for each $r=1, \ldots, R$, by a degree $d_{r}$ with which $r$-th rule is fired, we mean a number $d_{r}=$ $f_{\&}\left(\mu_{r 1}\left(z_{1}\right), \ldots, \mu_{r N}\left(z_{N}\right)\right)$.

- We say that a rule base is consistent if

$$
d_{1}+\ldots+d_{R} \leq 1 .
$$

For a consistent rule base, the degree $d_{0}$ with which the "else" rule is fired is defined as $d_{0}=$ $1-\left(d_{1}+\ldots+d_{R}\right)$.

- By a function $t=f_{\mathcal{R}}\left(z_{1}, \ldots, z_{N}\right)$ corresponding to the rule base $\mathcal{R}$, we mean a function

$$
t=d_{1} \cdot t^{(1)}+\ldots+d_{R} \cdot t^{(R)}+d_{0} \cdot t^{(0)} .
$$

\subsection{Resulting Description of a Two-Stage Control}

Definition 3. Let $n$ and $m$ be positive integers, let $x_{1}, \ldots, x_{n}$ be input variables, let $y_{1}, \ldots, y_{m}$ be auxiliary variables, and let $u$ be the control.

- By a two-stage rule base which describes $u$ in terms of $x_{i}$, we mean the collection consisting of $m$ rules bases $\mathcal{R}_{1}, \ldots, \mathcal{R}_{m}$ which describe $y_{1}, \ldots, y_{m}$ in terms of $x_{i}$, and a rule base $\mathcal{R}$ which describes $u$ in terms of $y_{1}, \ldots, y_{m}$.

- By a function $t=f\left(x_{1}, \ldots, x_{n}\right)$ corresponding to the two-stage rule base, we mean a function

$$
u=f_{\mathcal{R}}\left(y_{1}, \ldots, y_{m}\right)
$$

where, for every $j$ from 1 to $m$,

$$
y_{j}=f_{\mathcal{R}_{j}}\left(x_{1}, \ldots, x_{n}\right)
$$

\section{Main Result}

Theorem. Let $B=\left[a_{1}, b_{1}\right] \times \ldots \times\left[a_{n}, b_{n}\right]$ be an arbitrary $n$-dimensional box, let $f\left(x_{1}, \ldots, x_{n}\right)$ be a continuous function on this box, and let $f_{\&}$ be a continuous t-norm. Then, there exists a consistent two-stage rule base for which the corresponding function is exactly the given function $f\left(x_{1}, \ldots, x_{n}\right)$.

In other words, for every continuous function $f\left(x_{1}, \ldots, x_{n}\right)$ on a bounded set $B$, there exists a system of fuzzy rules which leads, after the two-stage defuzzification, exactly to this function.

In short, every possible control strategy can be not only approximated by a traditional one-stage fuzzy controller, it can also be exactly represented by a two-stage fuzzy controller. Since the two-stage representation is exact, the required number of rules does not depend on the approximation accuracy. So, the proposed approach shows promise in curtailing the rule base explosion. 
Comments.

- For the case of a single input variable $(n=1)$, a similar result was proven in $[1,4,14,20,21$, $30,31]$ : by using only two rules, we can exactly represent an arbitrary continuous function $f\left(x_{1}\right)$ on an interval $\left[a_{1}, b_{1}\right]$.

- The downside of this result is that while the standard approximation results use, say, triangular membership functions that are easy to store and to process, this new scheme uses special membership functions (which, in essence, code the desired control strategy). So, while we get fewer rules, we do not automatically decrease the total amount of information that needs to be stored.

\section{Proof}

\subsection{The Main Result on Which This Proof is Based}

The proof of our theorem is based on a theorem proven by A. Kolmogorov [11] as a solution to the conjecture of D. Hilbert, formulated as the thirteenth problem [7]: one of 23 problems that Hilbert has proposed in 1900 as a challenge to the XX century mathematics.

This theorem says that an arbitrary continuous function $f\left(x_{1}, \ldots, x_{n}\right)$ on an $n$-dimensional box (of arbitrary dimension $n$ ) can be represented as a superposition of addition and functions of one variable.

The beginning of this topic can be traced to the Babylonians, who found (see, e.g., [3]) that the solutions $x$ of quadratic equations $a \cdot x^{2}+b \cdot x+c=0$ (viewed as function of three variables $a, b$, and $c$ ) can be represented as superpositions of functions of one and two variables, namely, arithmetic operations and square roots. Much later, similar results were obtained for functions of five variables $a, b, c, d, e$, that represent the solution of quartic equations $a \cdot x^{4}+b \cdot x^{3}+c \cdot x^{2}+d \cdot x+e=0$. But then, E. Galois proved in 1830 that for higher order equations, we cannot have such a representation. This negative result has caused Hilbert to conjecture that not all functions of several variables can be represented by functions of two or fewer variables. Hilbert's conjecture was refuted by A. Kolmogorov and his student V. Arnold.

This result has already been used in soft computing: in 1987 , it was used to produce the first proof that neural networks are universal approximators [6] (see also $[18,19])$; later on, it was used to prove that an arbi- trary logical operation can be approximated by a combination of standard fuzzy logical operators (see, e.g., $[25,27])$.

It is worth mentioning that Kolmogorov's result is not only of theoretical value: it was used to speed up actual computations (see, e.g., [5, 24]).

\subsection{The Proof Itself}

In our proof, we will use a simplified (and improved) version of Kolmogorov's theorem, formulated in [28] (further improvements were later described in [29]):

Theorem. [28] For an arbitrary box

$$
B=\left[a_{1}, b_{1}\right] \times \ldots \times\left[a_{n}, b_{n}\right],
$$

every real continuous function $f: B \rightarrow R$ can be represented as

$$
f\left(x_{1}, \ldots, x_{n}\right)=\sum_{1 \leq j \leq 2 n+1} \chi\left(\sum_{1 \leq i \leq n} \psi_{j i}\left(x_{i}\right)\right),
$$

for some continuous functions $\chi(y)$ and $\psi_{j i}\left(x_{i}\right)$.

In other words, every continuous function $f\left(x_{1}, \ldots, x_{n}\right)$ on a box $B$ can be represented as

$$
f\left(x_{1}, \ldots, x_{n}\right)=\chi\left(y_{1}\right)+\ldots+\chi\left(y_{2 n+1}\right),
$$

where for every $j$ from 1 to $m=2 n+1$, we have

$$
y_{j}=\psi_{j 1}\left(x_{1}\right)+\ldots+\psi_{j n}\left(x_{n}\right) .
$$

In view of this result, one way to prove our theorem is to show that for each of the functions (7) and (8), we can find a one-stage rule base $\mathcal{R}$ (correspondingly, $\mathcal{R}_{j}$ ), to which this very function is corresponding. Then, by combining these one-stage rules bases $\mathcal{R}$ and $\mathcal{R}_{j}$, we will get the desired two-stage rule base which represents exactly the original function $f\left(x_{1}, \ldots, x_{n}\right)$.

We will show how this rule base $\mathcal{R}_{j}$ can be constructed for the function (8); for (7), the construction is similar. Since each function $\psi_{j i}\left(x_{i}\right)$ is a continuous function on the interval $\left[a_{i}, b_{i}\right]$, it has a finite minimum $m_{j i}$ and a finite maximum $M_{j i}$. To describe the value of this function in fuzzy terms, we construct the auxiliary membership function

$$
\mu_{j i}\left(x_{i}\right)=\frac{1}{n} \cdot \frac{\psi_{j i}\left(x_{i}\right)-m_{j i}}{M_{j i}-m_{j i}} .
$$

(This function is similar to an "optimization" membership function introduced by R. Bellman and L. Zadeh in [2].) Let $A_{j i}$ indicate the fuzzy set with the membership function $\mu_{j i}\left(x_{i}\right)$. We want to choose the real 
numbers $y_{j}^{(1)}, \ldots, y_{j}^{(n)}$ and $y_{j}^{(0)}$ in such a way that the rule base

$$
\begin{gathered}
\text { "if } A_{j i}\left(x_{i}\right) \text {, then } y_{j}=y_{j}^{(i)} \text { ", } 1 \leq i \leq n, \\
\text { "else } y_{j}=y_{j}^{(0)} \text { " }
\end{gathered}
$$

corresponds to the function (8). This rule base has $R=n$ regular rules and an "else" rule. For each $i$ from 1 to $n$, the degree to which $i$-th rule is fired is equal to $d_{i}=\mu_{j i}\left(x_{i}\right)$. Since $M_{j i}$ is a maximum of a function $\psi_{j i}\left(x_{i}\right)$, from our definition of $\mu_{j i}\left(x_{i}\right)$, it follows that $\psi_{j i}\left(x_{i}\right) \leq M_{j i}$ and therefore, that

$$
\psi_{j i}\left(x_{i}\right)-m_{j i} \leq M_{j i}-m_{j i}
$$

and $\mu_{j i}\left(x_{i}\right) \leq 1 / n$. Hence, $d_{i} \leq 1 / n$ for all $i$, and so $d_{1}+\ldots+d_{n} \leq 1 / n+\ldots+1 / n=1$; therefore, this rule base is consistent.

In accordance with Definition $2^{\prime}$, the function corresponding to this rule base is equal to

$$
\begin{gathered}
y_{j}=\sum_{i=1}^{n} y_{j}^{(i)} \cdot \mu_{j i}\left(x_{i}\right)+y^{(0)} \cdot\left(1-\sum_{i=1}^{n} \mu_{j i}\left(x_{i}\right)\right)= \\
\sum_{i=1}^{n}\left(y_{j}^{(i)}-y_{j}^{(0)}\right) \cdot \mu_{j i}\left(x_{i}\right)+y^{(0)} .
\end{gathered}
$$

Substituting the expression for $\mu_{j i}\left(x_{i}\right)$ into this formula, and separating terms proportional to $\psi_{j i}\left(x_{i}\right)$ from the terms which do not contain $\psi_{j i}$, we get the following formula:

$$
\begin{gathered}
y_{j}=\sum_{i=1}^{n} \frac{y_{j}^{(i)}-y_{j}^{(0)}}{n \cdot\left(M_{j i}-m_{j i}\right)} \cdot \psi_{j i}\left(x_{i}\right)+ \\
{\left[y^{(0)}-\sum_{i=1}^{n} \frac{\left(y_{j}^{(i)}-y_{j}^{(0)}\right) \cdot m_{j i}}{n \cdot\left(M_{j i}-m_{j i}\right)}\right] .}
\end{gathered}
$$

Thus, to get the formula (8), we must choose the values $y_{j}^{(i)}$ so that

$$
\begin{gathered}
y_{j}^{(i)}-y_{j}^{(0)}=n \cdot\left(M_{j i}-m_{j i}\right), \quad 1 \leq i \leq n, \\
y_{j}^{(0)}=\sum_{i=1}^{n} \frac{\left(y_{j}^{(i)}-y_{j}^{(0)}\right) \cdot m_{j i}}{n \cdot\left(M_{j i}-m_{j i}\right)} .
\end{gathered}
$$

Substituting (10) into (11), we conclude that

$$
y_{j}^{(0)}=\sum_{i=1}^{n} m_{j i}
$$

and therefore, from (10), that

$$
y_{j}^{(i)}=\sum_{i=1}^{n} m_{j i}+n \cdot\left(M_{j i}-m_{j i}\right)
$$

for $1 \leq i \leq n$. For these values $y_{j}^{(i)}$, the function (9) indeed coincides with (8).

So, each formula (8) can be represented by a one-stage rule base, and similarly, the formula (7) can be represented by a one-stage rule base. Hence, the original function $f\left(x_{1}, \ldots, x_{n}\right)$ can be represented by a twostage rule base. The theorem is proven.

\section{Acknowledgments}

This work was supported in part by NASA under cooperative agreement NCC5-209, by NSF grants No. DUE9750858 and CDA-9522207, by the United Space Alliance, grant No. NAS 9-20000 (PWO C0C67713A6), by the Future Aerospace Science and Technology Program (FAST) Center for Structural Integrity of Aerospace Systems, effort sponsored by the Air Force Office of Scientific Research, Air Force Materiel Command, USAF, under grant number F49620-95-1-0518, by the National Security Agency under Grant No. MDA904-98-1-0564, and by Hong Kong RGC grant $4138 / 97 \mathrm{E}$.

\section{References}

[1] P. Bauer, E. P. Klement, B. Moser, and A. Leikermoser, "Modeling of fuzzy functions by fuzzy controllers", In: H. T. Nguyen, M. Sugeno, R. Tong, and R. Yager (eds.), Theoretical aspects of fuzzy control, J. Wiley, N.Y., 1995.

[2] R. E. Bellman and L. A. Zadeh, "Decisionmaking in a fuzzy environment," Management Sci., 1970, Vol. 17, pp. B141-B164.

[3] C. B. Boyer and U. C. Merzbach, "A History of Mathematics", Wiley, N.Y., 1991.

[4] N. Foo, "New perspectives and old problems ...", Proceedings of the Soft Computing Symposium, University of New South Wales, 1994.

[5] H. L. Frisch, C. Borzi, G. Ord, J. K. Percus, and G. O. Williams, "Approximate Representation of Functions of Several Variables in Terms of Functions of One Variable", Physical Review Lett., 1989, Vol. 63, No. 9, pp. 927-929.

[6] R. Hecht-Nielsen, "Kolmogorov's Mapping Neural Network Existence Theorem", IEEE Int'l Conf. on Neural Networks, San Diego, 1987, Vol. 2, pp. 11-14.

[7] D. Hilbert, "Mathematical Problems, lecture delivered before the Int'l Congress of Mathematics in Paris in 1900," translated in Bull. Amer. Math, Soc., 1902, Vol. 8, pp. 437-479. 
[8] K. Hirota and M. Sugeno, "Industrial Applications of Fuzzy Technology in the World", World Scientific, Singapore, 1996.

[9] A. Kandel and G. Langholtz (Editors), "Fuzzy Control Systems", CRC Press, Boca Raton, FL, 1994.

[10] L. T. Kóczy and D. Tikk, "Approximation in rule bases", IPMU'96: Proceedings of the International Conference on Information Processing and Management of Uncertainty in Knowledge-Based Systems, Granada, July 1-5, 1996, pp. 489-494.

[11] A. N. Kolmogorov, "On the Representation of Continuous Functions of Several Variables by Superposition of Continuous Functions of One Variable and Addition", Dokl. Akad. Nauk SSSR, 1957, Vol. 114, pp. 369-373.

[12] B. Kosko, "Fuzzy Systems as Universal Approximators," IEEE Trans. on Computers, 1994, Vol. 43, No. 11, pp. 1329-1333.

[13] B. Kosko, "Optimal fuzzy rules cover extrema", International Journal of Intelligent Systems, 1995, Vol. 10, No. 2, pp. 249-255.

[14] B. Kosko, "Additive fuzzy systems: from function approximation to learning", In: C. H. Chen (ed.), Fuzzy Logic and Neural Network Handbook, McGrawHill, N.Y., 1996, pp. 9-1-9-22.

[15] B. Kosko and J. A. Dickerson, "Function approximation with additive fuzzy systems", Chapter 12 in: H. T. Nguyen, M. Sugeno, R. Tong, and R. Yager (eds.), Theoretical aspects of fuzzy control, J. Wiley, N.Y., 1995, pp. 313-347.

[16] V. Kreinovich, G. C. Mouzouris, and H. T. Nguyen, "Fuzzy rule based modeling as a universal control tool", In: H. T. Nguyen and M. Sugeno (eds.), "Fuzzy Systems: Modeling and Control", Kluwer, Boston, MA, 1998, pp. 135-195.

[17] V. Kreinovich, H. T. Nguyen, S. A. Starks, and Y. Yam, "Decision making based on satellite images: optimal fuzzy clustering approach", Proceedings of the 37th IEEE Conference on Decision and Control CDC'98, Tampa, Florida, December 16-18, 1998.

[18] V. Kurkova, "Kolmogorov's Theorem Is Relevant", Neural Computation, 1991, Vol. 3, pp. 617-622.

[19] V. Kurkova, "Kolmogorov's Theorem and Multilayer Neural Networks", Neural Networks, 1991, Vol. 5, pp. 501-506.

[20] J. Lee and S. Chae, "Completeness of fuzzy controller carrying a mapping $f: R \rightarrow R$ ", Proceedings of the IEEE International Conference on Fuzzy Systems FUZZ-IEEE'93, 1993, Vol. 1, pp. 231-235.
[21] J. Lee and S. Chae, "Analysis of function duplicating capabilities of fuzzy controllers", Fuzzy Sets and Systems, 1993, Vol. 56, pp. 127-143.

[22] A. Meystel, "Pilot level of a hierarchical controller for an unmanned mobile robot," IEEE J. of Robotics \& Automation, Vol. 4, No. 3, 1988, pp. 244-255.

[23] A. Meystel, "Knowledge Based Nested Hierarchical Control", in Advances in Automation and Robotics, Vol.2, Ed. G. Saridis, JAI Press, Greenwich, CT, 1990, pp. 63-152.

[24] M. Ness, "Approximative versions of Kolmogorov's superposition theorem, proved constructively", J. Comput. Appl. Math., 1993.

[25] H. T. Nguyen and V. Kreinovich, "Kolmogorov's Theorem and its impact on soft computing", In: R. R. Yager and J. Kacprzyk, "The Ordered Weighted Averaging Operators: Theory and Applications", Kluwer, Boston, MA, 1997, pp. 3-17.

[26] H. T. Nguyen and V. Kreinovich, "Methodology of fuzzy control: an introduction", In: H. T. Nguyen and M. Sugeno (eds.), "Fuzzy Systems: Modeling and Control", Kluwer, Boston, MA, 1998, pp. 19-62.

[27] H. T. Nguyen, V. Kreinovich, and D. Sprecher, "Normal forms for fuzzy logic - an application of Kolmogorov's theorem" International Journal on Uncertainty, Fuzziness, and Knowledge-Based Systems, 1996, Vol. 4, No. 4, pp. 331-349.

[28] D. A. Sprecher, "On the Structure of Continuous Functions of Several Variables", Transactions Amer. Math. Soc., 1965, Vol. 115, No. 3, pp. 340-355.

[29] D. A. Sprecher, "An Improvement in the Superposition Theorem of Kolmogorov", Journal of Mathematical Analysis and Applications, 1972, Vol. 38, pp. 208-213.

[30] F. A. Watkins, "Fuzzy Engineering", Ph.D. Dissertation, Department of Electrical and Computer Engineering, University of California at Irvine, 1994.

[31] F. A. Watkins, "The representation problem for additive fuzzy systems", Proceedings of the IEEE International Conference on Fuzzy Systems FUZZIEEE/IFES'95, March 1995, Vol. 1, pp. 117-122.

[32] Y. Yam, "Fuzzy Approximation via Grid Point Sampling and Singular Value Decomposition," IEEE Transactions on Systems, Man, and Cybernetics, 1997, Vol. 27, No. 6, pp. 933-951. 